\title{
A Microstructure-Guided Constitutive Modeling Approach for Random Heterogeneous Materials: Application to Structural Binders
}

\author{
Sumanta Das ${ }^{1}$, Amit Maroli $^{1}$, Sudhanshu S. Singh ${ }^{2, \#}$, Tyler Stannard ${ }^{2}$, Xianghui Xiao ${ }^{3}$, Nikhilesh \\ Chawla², Narayanan Neithalath ${ }^{1, *}$ \\ ${ }^{1}$ School of Sustainable Engineering and the Built Environment, Arizona State University, Tempe, AZ, \\ USA. \\ ${ }^{2}$ Materials Science and Engineering, Arizona State University, Tempe, AZ, USA \\ ${ }^{3}$ Advanced Photon Source, Argonne National Laboratory, Argonne, IL, USA \\ \# Current address, Department of Materials Science and Engineering, Indian Institute of Technology \\ Kanpur, Kanpur, UP, India
}

"Corresponding Author (Narayanan.Neithalath@asu.edu), Phone: 001-480-965-6023, Fax: 001-480-9650557

\begin{abstract}
This paper presents a microstructure-guided modeling approach to predict the effective elastic response of heterogeneous materials, and demonstrates its application towards two highly heterogeneous, unconventional structural binders, i.e., iron carbonate and fly ash geopolymer. Microstructural information from synchrotron $\mathrm{x}$-ray tomography (XRT) and intrinsic elastic properties of component solid phases from statistical nanoindentation are used as the primary inputs. The virtual periodic 3D microstructure reconstructed using XRT, along with periodic boundary conditions is used as a basis for strain-controlled numerical simulation scheme in the linear elastic range to predict the elastic modulus as well as the stresses in the microstructural phases. The elastic modulus of the composite material predicted from the microstructure-based constitutive modeling approach correlates very well with experimental measurements for both the materials considered. This technique efficiently links the microstructure to mechanical properties of interest and helps develop material design guidelines for novel heterogeneous composites.
\end{abstract}

Keywords: Microstructure; Constitutive model; Finite element; X-ray tomography; Nanoindentation; Binders 


\section{INTRODUCTION}

Design and development of novel heterogeneous materials requires a proper understanding of the influence of microstructure on the desired performance features. Structural materials are invariably designed based on mechanical performance, generally in the linear elastic limit. Accurate prediction of effective elastic properties of highly heterogeneous materials with multiple microstructural phases is a challenging task. This necessitates reliable classification and characterization of constituent phases in terms of their volume fractions, size distributions and intrinsic elastic properties, coupled with analytical or numerical models capable of handling more than just the volume fractions of the phases.

The effective elastic properties of heterogeneous materials have been predicted using effective media theories [1,2], self-consistent schemes [3-5], and various analytical homogenization methods [6-8]. However, in many cases, these methodologies only give estimates or bounds for the macroscopic properties. Furthermore, these techniques have been proved to be insufficient when the stiffness contrast between the phases is large or when large inclusion volume fractions are incorporated $[9,10]$. In the recent past, computational homogenization methods have been actively researched [11-15], with tremendous improvements achieved in terms of computational efficiency. Finite element method (FEM) is currently established as one of the most efficient numerical approaches to predict macroscopic behavior through microstructure-guided simulations [16-20]. This approach eliminates the shortcomings of the analytical schemes and thus yields a better solution [9]. However, the accuracy of predictions depend on the capability to adequately represent the microstructure both in terms of phase fractions, distributions and phase properties, the size of the RVE, and the choice of appropriate microscopic boundary conditions. The microstructure can be generated artificially using statistical information $[9,21,22]$ or actual 3D x-ray tomography images can be directly used $[9,23,24]$. While the former approach has been implemented in the past $[21,22]$, the direct use of $3 \mathrm{D}$ tomography information is rather computationally demanding. The voxel-based discretization approach used in conjunction with tomography images introduces distortions at the interfaces of the microstructural phases, thereby inducing artificial anisotropy, which necessitates significantly large number of elements to obtain a converged solution. Microstructure-based modeling also demands that appropriate microscopic boundary conditions be used. While commonly used uniform boundary conditions (Dirichlet or Neumann) requires larger RVEs to yield reasonable estimate of the effective property, periodic boundary conditions are effective with smaller RVEs even if the material does not demonstrate significant periodicity in its microstructure $[25,26]$. 
This paper presents a comprehensive microstructure-guided property prediction scheme for a large class of random heterogeneous materials by combining high-fidelity experimental techniques with finite element modeling, and validates it for two novel types of structural binders. The methodology involves: (1) generation of RVE of the heterogeneous material using a microstructural stochastic packing algorithm with inputs from actual microstructural features that are extracted from 3D synchrotron x-ray tomography, (2) assignment of local elastic properties obtained from statistical nanoindentation to the microstructural phases, (3) application of periodic boundary conditions in the RVE and FE analysis to simulate a strain-controlled test-scenario in the linear elastic regime (which is used in design and in the determination of in-service structural performance), and (4) determination of effective volume-averaged RVE stresses and strains and effective homogenized Young's modulus of the material through a homogenization module. The versatility of this approach is verified on two highly heterogeneous binder systems: (i) a novel iron carbonate binder [27-31] and (ii) a fly ash geopolymer $[8,32,33]$. Thus this study intends to demonstrate a numerical approach that can be used in conjunction with reliable microstructural descriptors, thereby enabling adequate prediction of the macroscopic elastic response of several heterogeneous materials, which in-turn facilitates microstructure-guided material design.

\section{MICROSTRUCTURE GENERATION AND THE MODELING SCHEME}

This section describes the microstructure-guided constitutive modeling framework for heterogeneous materials. The framework explained herein executes multi-scale analysis of heterogeneous materials involving generation of a unit cell based on known inclusion size distributions, meshing of the unit cell and application of appropriate boundary conditions, and microstructural stress analysis. The detailed procedure is explained in the following sub-sections.

\subsection{Generation of Representative Volume Element (RVE)}

Generation of RVE is accomplished here using the Lubachhevsky-Stillinger algorithm [34-37]. This algorithm employs non-overlapping particles on a cubical RVE. First, the desired number of particles are randomly distributed inside the periodic bounding box with random initial velocities of the particles. The radius of each particle is initialized as zero. The radius of $i^{\text {th }}$ particle $\left(r_{i}\right)$ in the next event is a function of the growth rate $\left(\mathrm{g}_{\mathrm{i}}\right)$, which is tailored to attain the desired particle size distribution, as shown in Eq. 1.

$$
\frac{d r_{i}}{d t}=g_{i}
$$

The growth rate between time $t^{n}$ and $t^{n+1}$ is computed using a finite difference scheme as follows: 


$$
g_{i}=\frac{\left(r_{i}^{n+1}-r_{i}^{n}\right)}{\Delta t}
$$

The particle radii are then updated using the growth rate and time increment. The position of particle "i" is also updated considering a constant velocity between time nodes. Checks for particle contacts and postcontact velocities are made in each time step, and all particle positions are updated using a forward Euler scheme. These steps are repeated, and in the process of iterations the particles change position in the bounding box, collide and grow in order to obtain the desired volume fraction. Finally, the obtained microstructural information is scripted in python language to be imported to $A B A Q U S^{\mathrm{TM}}$ for finite element implementation. More details on the microstructure generation algorithm can be found in [34-37].

\subsection{Boundary Conditions}

Choosing appropriate microscopic boundary conditions is an essential step in any numerical stress analysis procedure. In micromechanics, the commonly adopted boundary conditions are: essential boundary conditions where uniform displacements are applied at the boundaries, or Neumann boundary conditions where uniform tractions are prescribed at the boundaries of the unit cell. These boundary conditions have been applied to predict effective properties of several materials including cementitious binders [38-40]. Since it is difficult to realize uniform boundary conditions in experimental setups, mixed boundary conditions are also proposed $[41,42]$. In this work, periodic boundary condition is adopted since it has been shown to provide better approximations of effective properties of heterogeneous materials even with relatively smaller RVEs that are favorable for computational expediency $[25,26]$.

Periodic boundary condition ensures two continuity criteria at the boundaries of neighboring unit cells in order to ensure assembly of individual unit cells as a physical continuous body [43]: (i) displacement continuity, i.e., neighboring unit cells cannot be separated or they cannot penetrate each other; and (ii) traction continuity at the boundary of neighboring unit cells. The displacement field in any 2D periodic microstructure is given as:

$$
v_{i}\left(x_{1}, x_{2}\right)=\varepsilon_{i j}^{0} x_{j}+v_{i}^{*}\left(x_{1}, x_{2}\right)
$$

Here, $\varepsilon_{i j}^{0}$ is the applied strain tensor, and $v_{i}^{*}$ is a periodic function representing the modification of linear displacement field due to the heterogeneous microstructure. Figure 1 shows a schematic representation of periodic boundary conditions. For the unit cell shown in Figure 1(b), the displacements on a pair of parallel opposite boundary edges are given as: 


$$
\begin{aligned}
& v_{i}^{s+}=\varepsilon_{i j}^{0} x_{j}^{s+}+v_{i}^{*} \\
& v_{i}^{s-}=\varepsilon_{i j}^{0} x_{j}^{s-}+v_{i}^{*}
\end{aligned}
$$

Here, $s^{+}$and $s^{-}$are $s^{\text {th }}$ pair of two opposite parallel boundary surfaces of the unit cell. The periodic function $v^{*}$ is the same at both the parallel opposite edges due to periodicity. The difference between the displacement fields of the two opposite parallel boundary edges is given as:

$$
v_{i}^{s+}-v_{i}^{s-}=\varepsilon_{i j}^{0}\left(x_{j}^{s+}-x_{j}^{s-}\right)=\varepsilon_{i j}^{0} \Delta x_{j}^{s}
$$

For a pair of opposite parallel boundary edges, $\Delta x_{j}^{s}$ is constant for a specified $\varepsilon_{i j}^{0}$. The perturbation is introduced into the system of equations through a reference node which only acts as a carrier for the load and is not attached to any element in the model. The general form of complete set of equations can be written as:

$$
v_{i}^{s+}-v_{i}^{s-}+v_{i}^{\text {dummy }}=0
$$

As an example, to apply a compressive displacement in the 2-direction in the 3D RVE (see Figure 1 (c)), a linear constraint can be established between the reference node, bottom face (BF) node sets and top face (TF) node sets in the 2-direction as:

$$
u_{1}^{B F}-u_{1}^{T F}=0 ; u_{2}^{B F}-u_{2}^{T F}+u_{2}^{d u m m y}=0 ; u_{3}^{B F}-u_{3}^{T F}=0
$$

Linear equations of the above form are implemented for all parallel face pairs for complete implementation of periodic boundary condition in 3D RVE. These sets of linear constraint equations are appended to the previously generated python language script containing microstructural information. A specific strain is implemented through the constraint equations to simulate a strain-controlled test scenario. More details on application of periodic boundary condition can be found in $[26,44,45]$. 


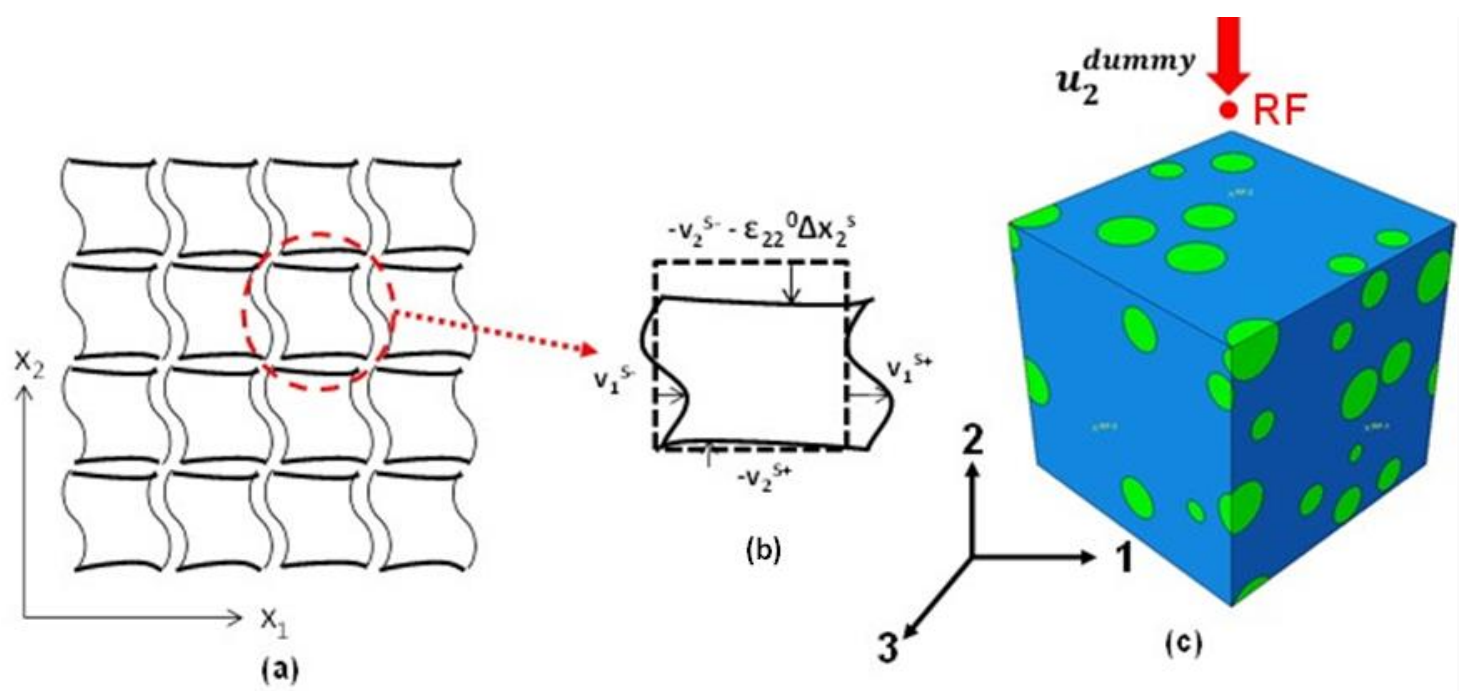

Figure 1: Schematic representative element area (REA) under applied strain $\left(\varepsilon_{22}^{0}\right)$ with periodic boundary conditions: (a) repetitive periodic unit cells; (b) 2D REA showing periodic boundary conditions; and (c) periodic boundary condition implemented in a 3D RVE for a two-phase composite.

\subsection{Finite Element Simulation and Numerical Homogenization}

The RVE is meshed through the python script and the analysis is implemented using ABAQUS ${ }^{\mathrm{TM}}$ solver. A strain- (or displacement-) controlled test on the RVE is simulated. To efficiently handle post-processing of the simulated individual element stresses, a homogenization module is implemented to obtain effective volume-averaged RVE stresses and strains [46]. The post-processing module contains a Matlab subroutine that operates on the data file generated by ABAQUS ${ }^{\mathrm{TM}}$ containing elemental volumes and average elemental stresses/strains, and yields volume-averaged stresses/strains. The effective stresses are computed based on the different applied strains, thereby facilitating the development of an effective constitutive relationship in the linear elastic regime. Figure 2 depicts the flowchart for the analysis procedure. 


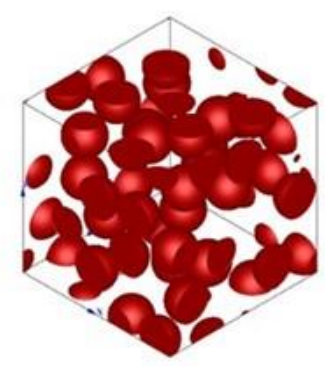

Generation of microstructure

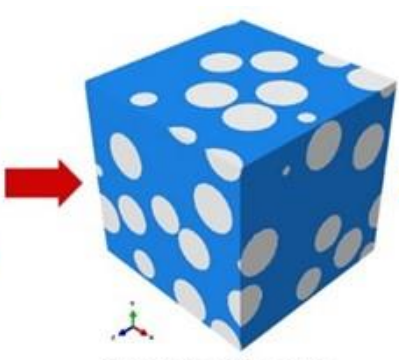

3D microstructure (Python script for ABAQUS solver

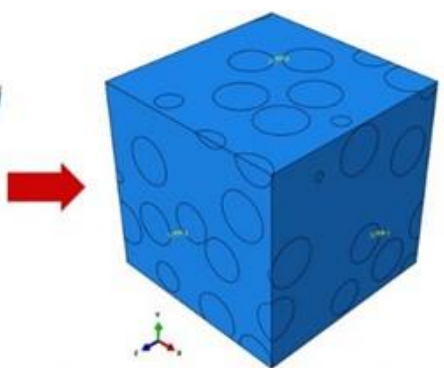

Periodic Boundary Conditions

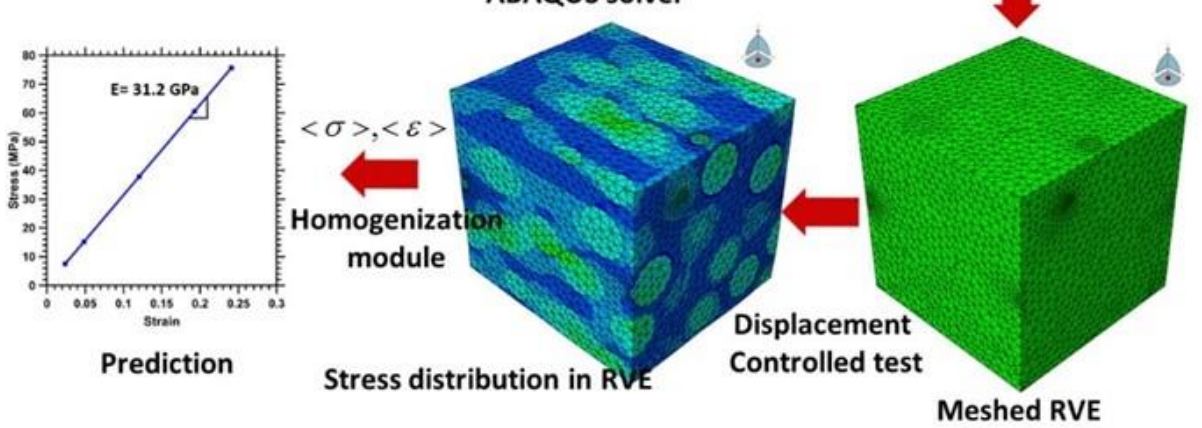

Figure 2: Numerical analysis sequence showing the microstructural generation, application of periodic boundary conditions, meshing, homogenization, and determination of average RVE stresses and effective $E$.

\section{EXPERIMENTAL PROGRAM}

In the remainder of this paper, the prediction scheme explained in the previous sections is applied to two different heterogeneous binders - iron carbonate and fly ash geopolymer. Figure 3 shows scanning electron micrographs depicting the heterogeneity in these binders. Figure 3(a) shows a representative backscattered SEM micrograph for the iron carbonate binder showing the unreacted iron particles (white), pores (dark phase) and the continuous matrix. The matrix shows significant variation in grey scale values implying heterogeneity in phase stiffness. Characterization of different solid phases present in matrix is accomplished using synchrotron tomography and nanoindentation as explained later in this section. Figure 3(b) shows a micrograph of the fly ash geopolymer. These micrographs demonstrate the presence of unreacted/partially reacted fly ash (rounded particles), pore phase and the matrix (N-A-S-H gel [32]) in the fly ash-based geopolymer. The spatial greyscale variation in matrix is significantly lower as compared to that observed in iron carbonate binder. This section describes the synthesis of these binders and the experimental tools used to characterize them. The microstructural features and their intrinsic nanoscale mechanical properties are used to predict macro-scale mechanical response of these materials. 


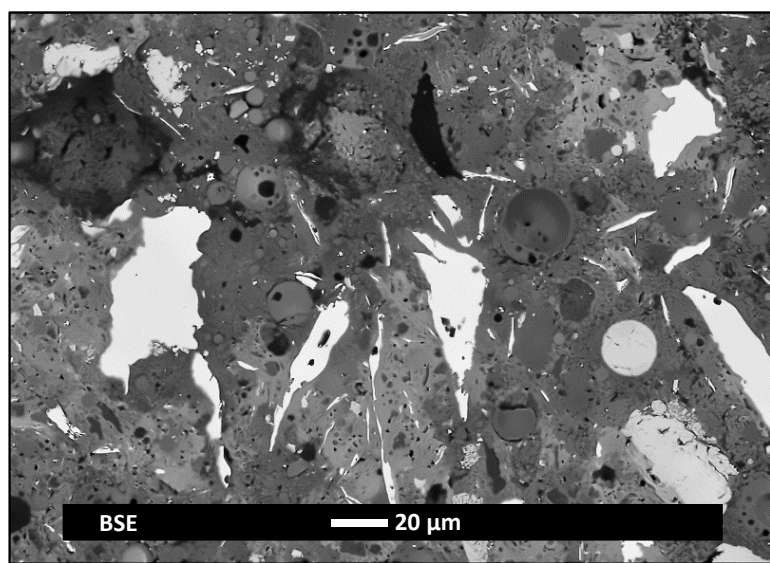

(a)

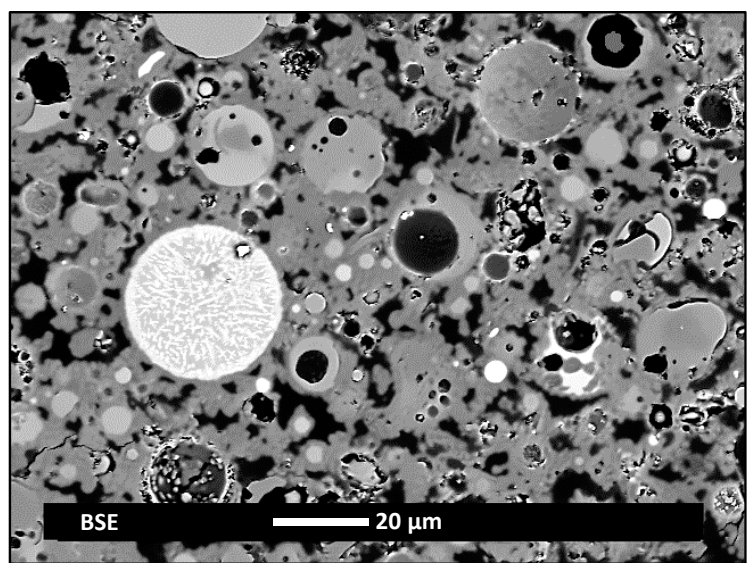

(b)

Figure 3: Representative backscattered SEM micrographs depicting the heterogeneity in: (a) iron carbonate binder; (b) alkali-activated fly ash geopolymer

\subsection{Materials and Mixture Proportions}

For the synthesis of iron carbonate binder, metallic iron powder with a median particle size of $19 \mu \mathrm{m}$ was used as the major starting material. The other additives used in the binder formation included Class F fly ash and metakaolin conforming to ASTM C 618, limestone powder (median particle size of $0.7 \mu \mathrm{m}$ ) conforming to ASTM C 568, and oxalic acid as an agent for iron dissociation. The rationale for the use of these additives and their influence on property development and microstructure have been detailed in $[27,29]$. Further details on the ingredients, mixture proportioning, and curing of this novel binder material can be found in [27]. The binder used in this study includes 60\% iron powder, $20 \%$ fly ash, $8 \%$ limestone, $10 \%$ metakaolin and $2 \%$ oxalic acid by mass. These proportions were arrived at based on strength and porosity of several trial mixtures [27]. Prismatic beams of size $127 \mathrm{~mm}$ (length) x $25.4 \mathrm{~mm}$ (depth) x 25.4 $\mathrm{mm}$ (width) were prepared in polycarbonate molds and the samples were placed inside clear plastic bags in a $100 \% \mathrm{CO}_{2}$ environment under normal temperature and pressure for 24 hours. The beams were then demolded and kept in a $100 \% \mathrm{CO}_{2}$ environment for 5 more days. After carbonation, the samples were placed in air for 4 days for evaporation of moisture from the samples before subjecting them to required testing.

For the fly ash geopolymer, a Class F fly ash conforming to ASTM C 618 was used as the starting material. $8 \mathrm{M} \mathrm{NaOH}$ solution was used to activate the fly ash. The alkaline solution was added to fly ash, and mixed for 4 minutes in a laboratory mixer. The mixtures were then filled in molds and subjected to heat curing in a laboratory oven at $60^{\circ} \mathrm{C}$ for 48 hours, in sealed conditions. More details can be found in [32]. 


\subsection{Synchrotron X-ray Tomography}

Synchrotron X-ray tomography (XRT) [47] was performed at the 2-BM beamline of the Advanced Photon Source (APS) at Argonne National Laboratory. Details of APS beamline 2-BM have been described elsewhere $[48,49]$. A monochromatic beam with energy of approximately $24 \mathrm{keV}$ was focused on a cylindrical specimen $9.8 \mathrm{~mm}$ diameter and $8.5 \mathrm{~mm}$ long. The transmitted $\mathrm{x}$-rays were converted to visible light using a LUAG:Ce scintillator screen coupled with an objective lens and CoolSnap K4 CCD camera to achieve a resolution of $0.74 \mu \mathrm{m} /$ pixel and $0.65 \mu \mathrm{m} /$ pixel respectively for the fly ash geopolymer and iron carbonate binders. 2D projections were acquired at angular increments of $0.12^{\circ}$ over a range of $180^{\circ}$. The 2D projections were then reconstructed using a Fast Fourier Transform (FFT)-based Gridrec algorithm [50-52]. Synchrotron microtomography has been shown to be highly suitable for the evaluation of pore structure that is influential in dictating the bulk mechanical behavior of the binders [53-56].

\subsection{Nanoindentation}

For nanoindentation tests, cubic pieces from the iron carbonate and fly ash geopolymer binders, having a side length of $4 \mathrm{~mm}$ were used. The smaller specimens were removed from the interior of larger prismatic specimens prepared for macroscale tests. The samples were ultrasonically cleaned, rinsed with isopropyl alcohol and dried to remove debris from sectioning/handling. The samples were then encapsulated using a two-part epoxy followed by vacuum impregnation at $95 \mathrm{KPa}$ and overnight curing at room temperature. Samples were polished using silicon carbide ( $\mathrm{SiC}$ ) paper. Successive polishing steps were carried out using smaller sized abrasives, and completed using a $0.04 \mu \mathrm{m}$ colloidal silica suspension to ensure a smooth surface.

The nanoindentation measurements were carried out on the polished sample in a commercial nanoindenter (Nanoindenter XP-II, Agilent) using a Berkovich tip (three-sided pyramid indenter). Indentations were carried out at initially at $10 \mu \mathrm{m}$ grid spacing on an area approximately $250 \mu \mathrm{m} \times 250$ $\mu \mathrm{m}$, in size which is considered representative for cementitious materials [53,57]. All the indentation locations were carefully selected through optical microscopy prior to testing to ensure that pores or cavities are not encountered in the process. Thus, the indentation experiments were carried out mostly on the solid phases, which resulted in no spurious peaks in the frequency distribution curves of the elastic modulus. Continuous stiffness measurement (CSM) technique [58-60] was employed to measure the Young's modulus of phases. A constant Poisson's ratio of 0.20 is used for all the phases other than the unreacted iron particles ( $v=0.30$ for iron particles) since the effect of variation of Poisson's ratio in the 
range of 0.18 to 0.22 has been reported to be insignificant in the calculation of Young's modulus for similar systems $[61,62]$.

\subsection{Scanning Electron Microscopy (SEM)}

Plane polished samples were prepared in a similar manner as for nanoindentation experiments. The samples were imaged under a Philips XL30 Field Emission Environmental Scanning Electron Microscope (FESEM) in the backscattered mode to obtain high resolution micrographs.

\subsection{Displacement-Controlled Flexural Test on Beams}

Three-point bend tests were performed on prismatic beams of size $127 \mathrm{~mm}$ (length) x $25.4 \mathrm{~mm}$ (depth) $\mathrm{x}$ $25.4 \mathrm{~mm}$ (width) (span of $101.6 \mathrm{~mm}$ ). Six replicate beams were tested. The beams were tested in a midspan displacement-controlled mode at a constant displacement rate of $0.004 \mathrm{~mm} / \mathrm{sec}$. The mid-span displacement was measured continuously using a linear variable displacement transducer (LVDT). The test was terminated when the load reached about $80 \%$ of the peak load which was determined previously from simple flexural tests.

\section{APPLICATION OF THE MODEL TO MULTI-PHASE HETEROGENEOUS BINDERS}

This section describes the application of the microstructure-guided micromechanical model to evaluate the microstructural stresses and elastic response of the iron carbonate and fly ash geopolymer binders. The pore and microstructural features extracted from synchrotron $\mathrm{x}$-ray tomography and the nanomechanical phase properties from statistical nanoindentation are used in the models.

\subsection{Iron Carbonate Binder}

\subsubsection{D Characterization and quantification of component phases using Synchrotron X-Ray Tomography (XRT)}

For XRT-based microstructural quantification, several large cubic volumes of interest (up to $400^{3}$ voxels) are extracted from different areas of specimen subjected to XRT. The segmentation of the images involves selection of threshold greyscale values for different component phases in order to compute their volume fractions and size distributions. In heterogeneous materials, segmentation of all the component phases with minimal greyscale contrast is difficult $[8,53]$. For iron carbonate binder, the difficulty increases because of presence of large number of component phases with minimal greyscale contrast. Hence, in this study only the pore and unreacted iron particles (including unreacted zones in a partly reacted particle) are quantified from XRT since these phases exhibit significant greyscale contrast for accurate segmentation. The segmentation was implemented using Avizo Fire $^{\mathrm{TM}}$ software package. A transition point 
(in the grey scale histogram)-based segmentation process $[53,55]$ was employed where a small increment in the threshold values causes a sharp change in the detected phase quantities.

Figure 4(a) shows an original representative cubic volume of interest of size $300^{3}$ voxel $\left(260^{3} \mu \mathrm{m}^{3}\right)$ and Figure 4(b) shows the segmented unreacted iron particles. Figure 4(c) shows the pore, unreacted iron and solid phases segmented using the transition point-based segmentation process. Since it is difficult to quantify the different solid phases, in this study the use of synchrotron XRT data is only limited to quantification of unreacted iron particles and pores. Quantification of volume fractions of distinct solid phases other than pore and unreacted iron particles is carried out using frequency of occurrences of such phases obtained from statistical nanoindentation as presented in detail in a forthcoming section. Figures 5(a) and (b) show the size distributions of pores and iron particles. The segmented porosity and volume fraction of unreacted iron obtained from XRT are $25.8 \pm 0.5 \%$ and $13.4 \pm 0.4 \%$ respectively. The volume fractions and size distributions obtained from XRT are used in the microstructural model as explained later.

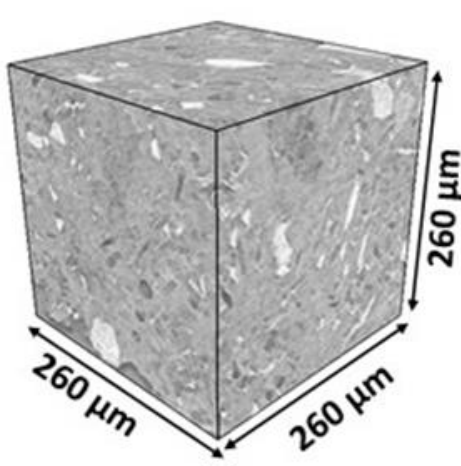

(a)

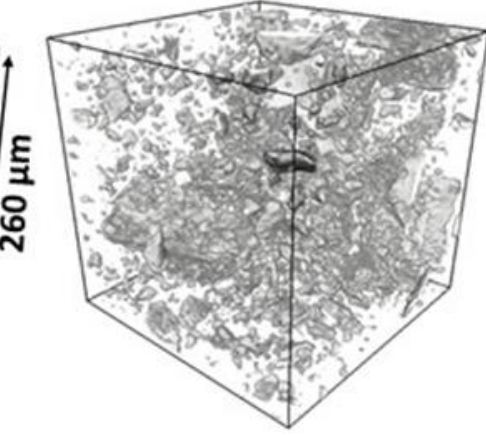

(b)

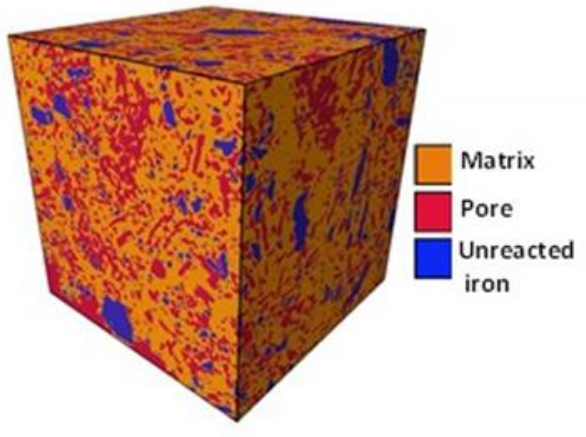

(c)

Figure 4: (a) Original XRT image of the iron carbonate binder, (b) 3D image after segmentation of unreacted iron particles; and (c) 3D image after pore and unreacted iron segmentation (three-phase; pore, unreacted iron and solid). Please refer to the online version of the paper for color images. 

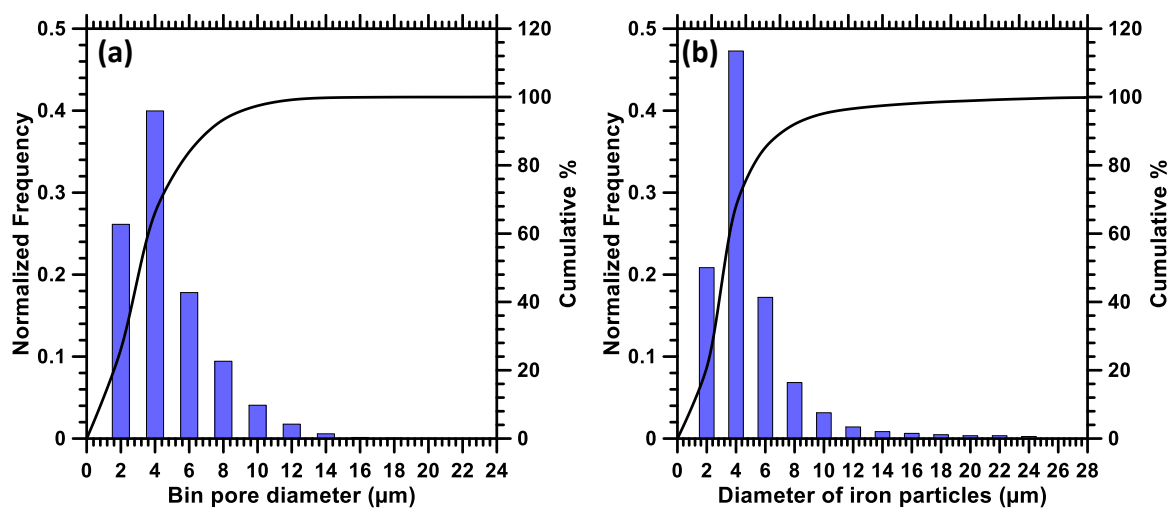

Figure 5: (a) Pore size distribution and (b) particle size distribution of iron in the iron carbonate binder from 3D XRT images

\subsubsection{Phase properties using nanoindentation}

The elastic properties of the individual phases in the binder system serve as inputs to the micromechanical analysis scheme for the determination of composite elastic properties. For classification of different solid phases in the iron-based binder and extraction of their elastic moduli, indentations were performed on a $250 \mu \mathrm{m} \times 250 \mu \mathrm{m}$ grid as described earlier, coupled with microscopic observations of the indentation locations to ensure proper classification and quantification of component phases. In order to avoid surface effects, penetration depths of up to $500 \mathrm{~nm}$ were employed. Figure 6(a) shows the representative loadpenetration depth plots corresponding to different solid phases in the microstructure. The process of identification of phases is described in a later part of this section. The computed Young's moduli of different phases as a function of penetration depth using the CSM technique are shown in Figure 6(b). Average Young's modulus in a penetration depth range of 200 -to-300 nm (where the modulus is independent of depth) is used for further analysis. From experimental measurements, the probability density functions (PDFs) are obtained using a bin size of 5 GPa which is presented in Figure 6(c). Seven characteristic peaks are clearly visible in the histogram signifying the plausible presence of seven different solid phases in the microstructure. The individual elastic properties of the seven phases has been obtained from statistical deconvolution which involves random seeding using a Monte Carlo simulation and minimization of quadratic deviations between the experimental and theoretical PDFs to determine the relevant parameters of the Gaussian distribution functions [62-64]. Figure 6(c) also shows the deconvoluted PDFs for seven different solid phases. These phases are assigned based on visual observation of the indentation locations along with a prior understanding of the phases present in the microstructure of the iron-based binder. The deconvolution results of the seven peaks are shown in Table 1. 

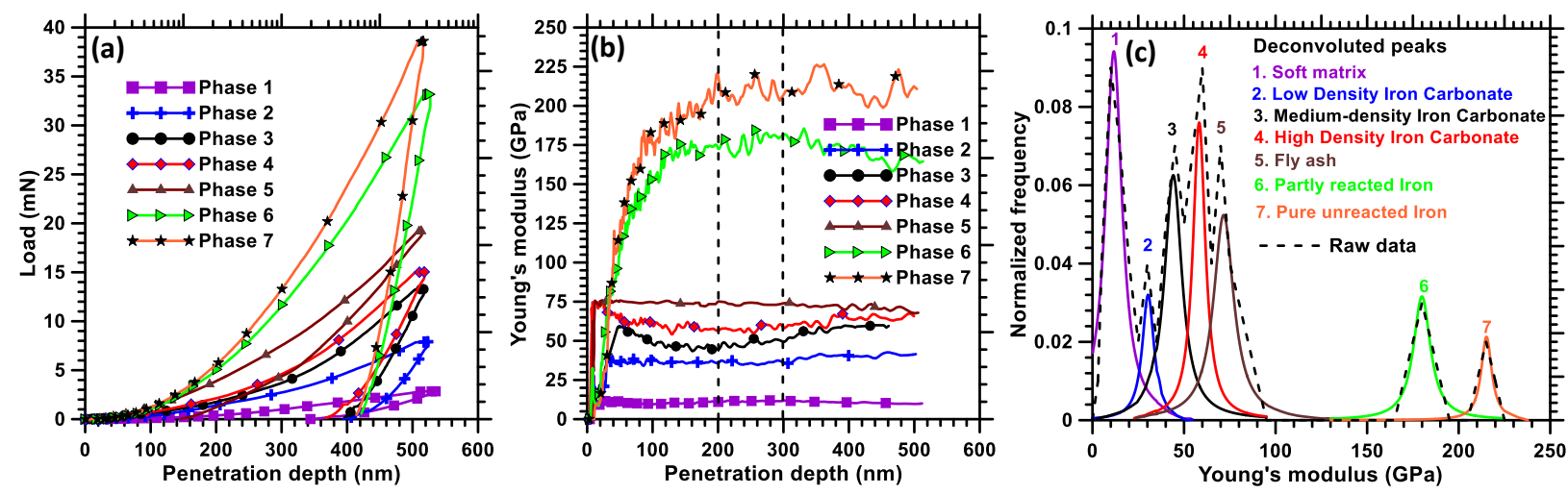

Figure 6: (a) Load-penetration depth curves for different phases in the iron-based binder, (b) elastic modulus as a function of the penetration depth for the distinct solid phases, and (c) deconvoluted component peaks for the seven distinctly identifiable microstructural phases in the solid component of the paste (the dashed line represents the raw data). The area under each deconvoluted peak is the fraction of that respective solid phase in all the solids in the heterogeneous binder.

Figure 7(a) shows the microstructure of the iron carbonate binder at different magnifications and the representative indentation locations that are used for the classification of phases. Locations A through $\mathrm{G}$ are marked in the first micrograph, followed by zoomed-in images in the following three micrographs in Figure 7(a). The corresponding phase elastic moduli are reported in Figure 7(b). The brightest phase in the microstructure are the unreacted iron particles. The indentation on this phase (Point D in Figure 7(a)) results in the maximum Young's modulus (Peak 7 in Figure 6(c)). Figure 6(c) also shows the indentation modulus results corresponding to iron particles that are oxidized and thus demonstrate a slightly lower elastic modulus (Peak 6). The peak 5 in Figure 6(c) corresponds to fly ash, denoted by the indentation location E in the microstructure (Figure 7(a)) and the measured Young's modulus value is shown in Figure 7(b). Peaks in the range of 25-to-60 GPa (Peaks 2, 3 and 4 in Figure 6(c), and indentation locations A, B, C, F, G in Figure 7(a)) correspond to iron carbonate phases that are formed through dissolution of iron into the matrix and its reaction with dissolved $\mathrm{CO}_{2}[27,29]$. The elastic moduli of iron carbonate phases decrease as the distance from the particle boundary increases as can be seen in Figures 7(a) and (b). Thus, three characteristic iron carbonate peaks (classified here for the sake of convenience as low density, medium density, and high density - LD, MD, and HD respectively) are obtained. The Young's modulus corresponding to Peak 1 in Figure 6(c) corresponds to the regions in the microstructure that mostly contains the reaction products from metakaolin and fine limestone powder, and hence is assigned as the soft matrix phase. 

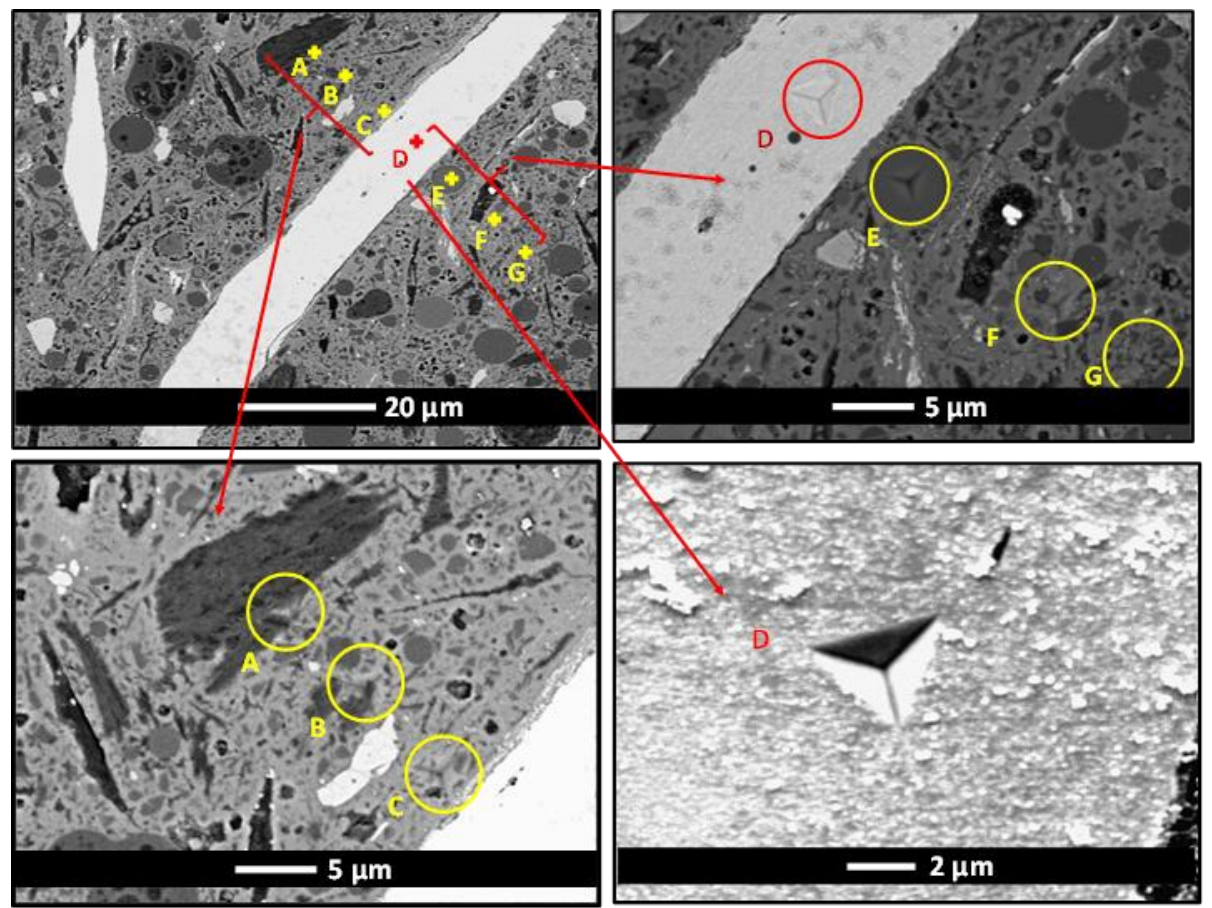

(a)

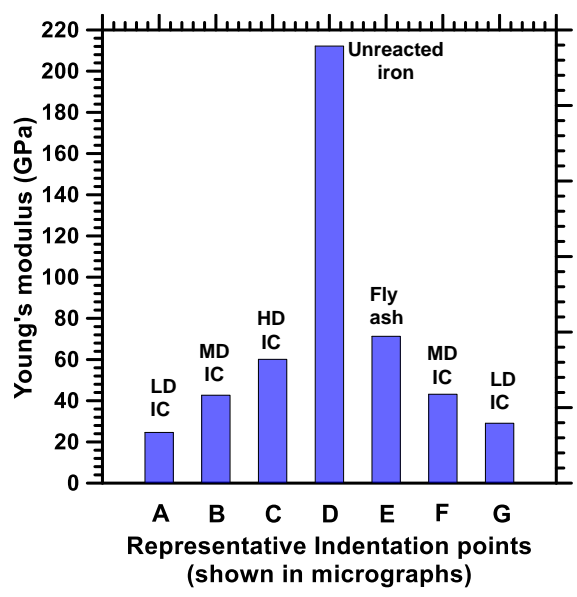

(b)

Figure 7: (a) Indentation locations as observed in SEM under backscattered mode, (b) Young's modulus values at the indentation locations shown in Figure 7(a). IC indicates iron carbonate. 
Table 1: Elastic properties of individual solid phases in iron-based binder and their frequency of occurrence in the microstructure as determined by statistical nanoindentation

\begin{tabular}{|c|c|c|c|c|}
\hline $\begin{array}{c}\text { Peak No. in } \\
\text { Fig. 6(c) }\end{array}$ & $\begin{array}{c}\text { Indentation } \\
\text { location in Fig. } \\
\text { 7(a) }\end{array}$ & Phases & E (GPa) & $\begin{array}{c}\text { Frequency of } \\
\text { occurrence }\end{array}$ \\
\hline 1 & -- & Soft matrix phase & $11.7 \pm 4.9$ & $22.5 \%$ \\
\hline 2 & A, G & Low density (LD) Carbonate & $30.3 \pm 7.8$ & $6.0 \%$ \\
\hline 3 & B, F & Medium density (MD) Carbonate & $44.1 \pm 11.5$ & $18.0 \%$ \\
\hline 4 & C & High Density (HD) Carbonate & $58.23 \pm 8.3$ & $15.8 \%$ \\
\hline 5 & E & Fly ash & $71.7 \pm 13.5$ & $24.6 \%$ \\
\hline 6 & -- & Partially oxidized Iron & $179.9 \pm 11.4$ & $3.8 \%$ \\
\hline 7 & D & Pure Iron & $215.2 \pm 7.0$ & $9.3 \%$ \\
\hline
\end{tabular}

\subsubsection{Two-stage homogenization for RVE stress analysis and effective property prediction}

The microstructure-guided numerical homogenization approach presented earlier is applied here to determine the stresses in the microstructural phases and the RVE, and consequent prediction of the elastic modulus of the bulk material. As seen from Figure $7(b)$, the stiffness contrast between unreacted iron and the remaining phases in the microstructure is quite large; hence the numerical simulations will be employed on a three-phase composite - i.e., unreacted iron particles, matrix, and the pores. While the matrix consists of phases with differing elastic moduli as described before, it is homogenized for ease of analysis.

Initially, the two solid entities, i.e., the iron particles and the matrix need to be simulated, followed by the inclusion of the pores. The matrix is quite complex as observed from Figures 6 and 7. Since the binder contains seven solid phases (Table 1), the phases with lower stiffness contrast such as the LD, MD, and HD iron carbonates, the soft matrix phase and fly ash particles are sequentially homogenized using analytical models. This initial homogenization sequence consists of the following steps: (i) HD iron carbonate phase homogenized with the MD phase; (ii) LD phase homogenized with the E obtained from the previous step to obtain the composite $\mathrm{E}$ of the iron carbonate reaction products; (iii) soft matrix phase then homogenized with the resultant from Step ii; and (iv) fly ash inclusions homogenized into the resultant matrix phase from Step iii to obtain the composite E of the matrix. Here, Steps (i) to (iii) are performed using relative frequency of occurrences of the solid phases and the corresponding phase Young's moduli obtained from nanoindentation (Table 1) by applying the Reuss and Voigt models (upper and lower bounds) [65-67] and averaging the results. For Step iv, the Mori-Tanaka model [6] which 
considers unreacted fly ash to be embedded in an infinitely extended matrix (the homogenized medium from Step iii) is implemented. This four-step process uses a combination of frequency of occurrences of phases and phase elastic properties obtained from nanoindentation to obtain the homogenized elastic modulus of the matrix phase in the binder. A similar analytical procedure can be employed for any multiphase heterogeneous material to minimize the number of effective microstructural phases needed for the computational simulations and thus the computational expense.

The numerical homogenization for the effective $E$ of the iron carbonate binder is performed here in two stages: (1) Stage-l: homogenization of unreacted iron inclusions into the matrix (from the above mentioned four-step process), and (2) Stage-II: homogenization of pores into the system from Stage-I. This approach is designed to reduce the computational demand since consideration of all three phases together is not computationally expedient when reasonable microstructural sizes are used. Figure 8(a) shows the 3D FE model of the Stage-1 microstructure generated using the approach presented earlier. Size distributions for iron particles, obtained from XRT (Figure $5(b)$ ), are used in the microstructure generation. Spherical particles are used for computational expediency here, and is justified by the fact that the results from using ellipsoidal particles were not significantly different. The 3D unit cell has a size of $75^{3} \mu \mathrm{m}^{3}$. The size of the representative unit cell was arrived at based on insignificant improvement of the homogenized result when larger unit cells $\left(>100^{3} \mu \mathrm{m}^{3}\right)$ are considered. The generated microstructure was implemented in a Python language script and imported into ABAQUS ${ }^{\mathrm{TM}}$. The periodic boundary conditions were implemented on the 3D unit cell as nodal displacement constraints (Refer to Section 2.2). The unit cell was meshed using tetrahedral elements (C3D10 element in ABAQUS ${ }^{\mathrm{TM}}$ ). A mesh convergence study was conducted to establish the mesh size for FE analysis. For the unit cell considered here, the finest mesh that yielded a converged solution contains 868989 nodes and 630014 elements and is used in further simulations (where varying strains are imposed in the RVE). Figure 8(b) shows the dominant principal stress $\left(\sigma_{22}\right)$ distribution in the unit cell corresponding to an applied strain of $0.12 \%$. The stiffer iron particles are locations of stress-concentrations. The volume-averaged principal stresses and strains, obtained using the post-processing module, corresponding to different externally applied strains in the linear elastic regime are shown in Figure 8(c). The elastic modulus of the Stage-I composite containing unreacted iron particles and the matrix is $46 \mathrm{GPa}$. 


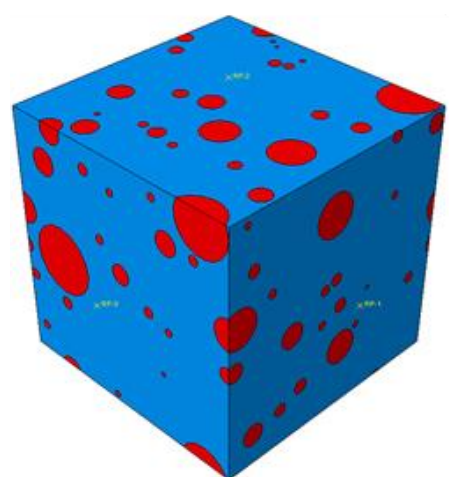

(a)

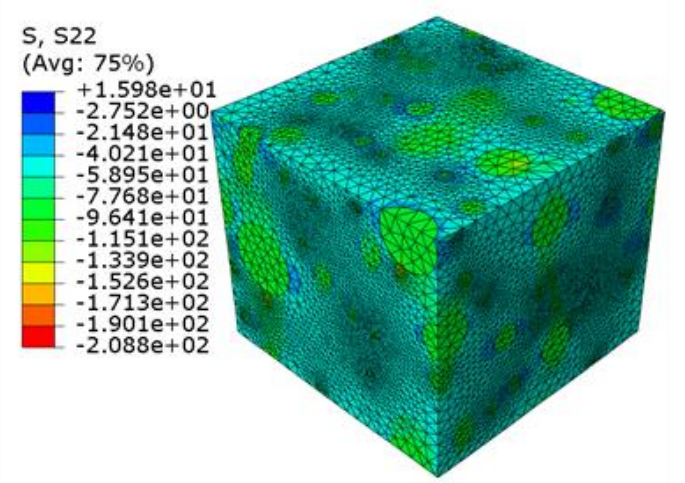

(b)

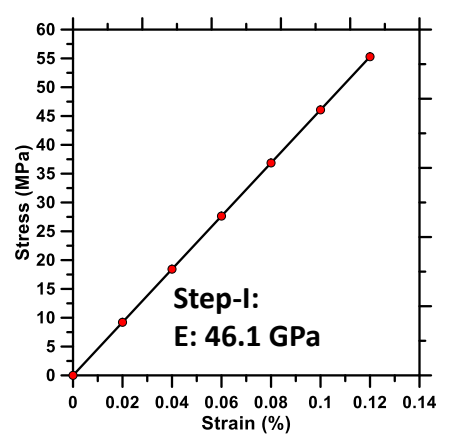

(c)

Figure 8: Stage-I: (a) FE model showing the unreacted iron inclusions and matrix; (b) stress distribution $(\mathrm{MPa})$ under an imposed strain of $0.12 \%$ which results in an average RVE stress of $55.2 \mathrm{MPa}$, and (c) the constitutive relationship in the linear elastic regime.

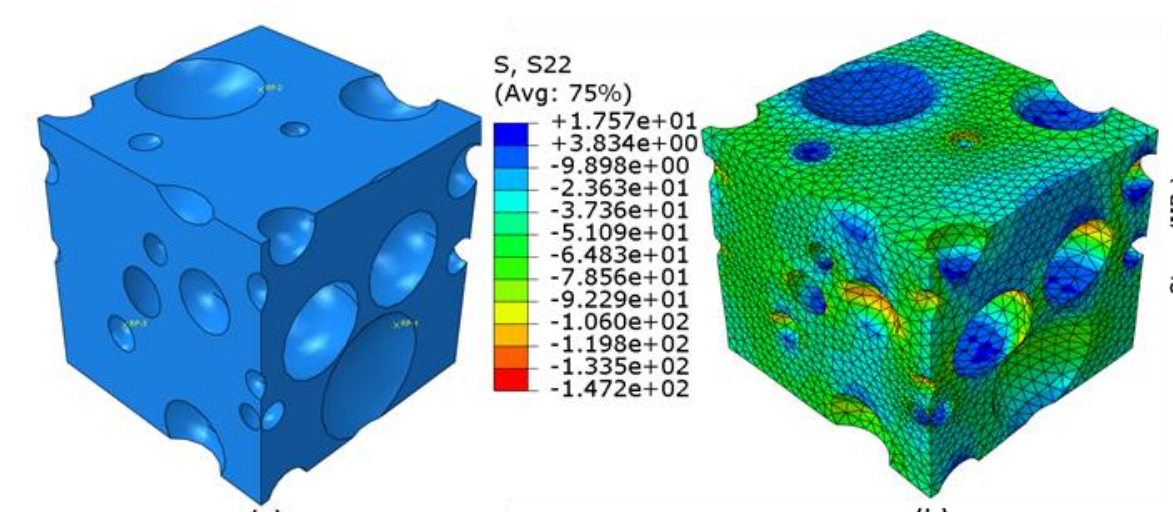

(a) (b)

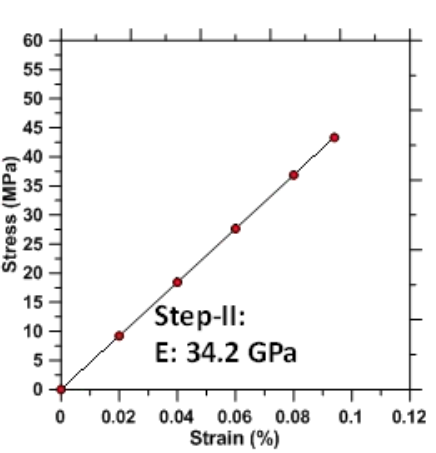

(c)

Figure 9: Stage-II: (a) FE model showing the matrix (from Stage-I - Figure 8) and the pores ; (b) stress distribution (MPa) under an imposed strain of $0.1 \%$ which results in an average RVE stress of $34.2 \mathrm{MPa}$, and $(c)$ the constitutive relationship in the linear elastic regime

Stage-II is implemented in such a way that that the matrix now has the homogenized elastic property obtained from Stage-I. The pore structure is generated based on actual pore size distributions obtained from XRT (Figure 5 (a)). The 3D unit cell has a pore volume fraction of $25.8 \%$ as determined from XRT. Figure 9(a) shows the 3D unit cell containing the pores and the matrix. The dominant principal stress distribution in the unit cell corresponding to applied strain of $0.1 \%$ is shown in Figure 9 (b). The linear elastic constitutive relationship thus obtained after post-processing for different applied strains is shown in Figure 9(c). The homogenized Young's modulus of $34.2 \mathrm{GPa}$ obtained for the iron-based binder from the numerical simulations correlates very well with the macroscale experimental value (34.6 $\pm 0.9 \mathrm{GPa})$ obtained from three-point-bending tests on prismatic beams. 


\subsubsection{Comparison of predicted Young's modulus obtained from 2D and 3D models}

This section compares the elastic moduli obtained from two-stage 3D FE analysis and a single stage 2D FE analysis. For a 3D analysis, the computational demand increases when: (i) all three phases are considered together, and (ii) realistic iron particle shapes (ellipsoids) are incorporated. In a 2D analysis, both of these aspects can be incorporated efficiently. Figure 10(a) shows the generated 2D microstructure containing iron particles, pores and the homogenized matrix. The iron-particles are generated here as equivalent ellipsoids (with an aspect ratio of 2 , which is more in line with the physical reality). Free quad-dominated 4-noded bilinear plane strain quadrilateral elements (CPE4R element implemented in ABAQUS ${ }^{\mathrm{TM}}$ ) are used in the 2D FE model. Figure $10(\mathrm{~b})$ shows the dominant principal stress $\left(\sigma_{22}\right)$ distribution in the microstructure for an externally applied strain of $0.12 \%$. Figure 10 (c) shows the constitutive relationship obtained from 2D FE analysis and post-processing. It is observed that the 2D model yields a higher (by 10\%) homogenized Young's modulus as compared to that obtained from 3D model or the experiments. Even though the 2D model allows simultaneous consideration of multiple phases and near-realistic shape of iron particles, the 3D stress interactions are missing in the 2D model, resulting in over-estimation of the Young's modulus. It is thus noted that the two-stage homogenization approach and a subsequent 3D FE analysis yields a more accurate estimate of composite Young's modulus as compared to the single-step 2D approach. Similar observations have been reported in $[68,69]$. However it needs to be mentioned that, given the computational demand, the accuracy of results from the $2 \mathrm{D}$ analysis is quite respectable, rendering it as a tool for fast and efficient comparison of different material systems.

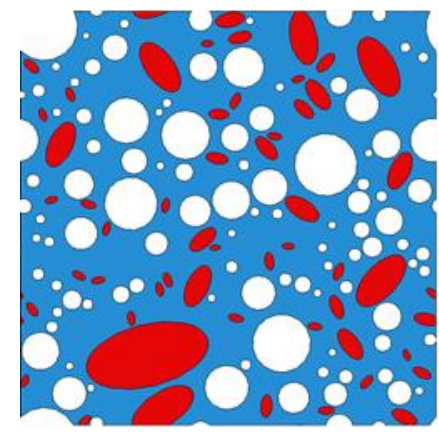

(a)
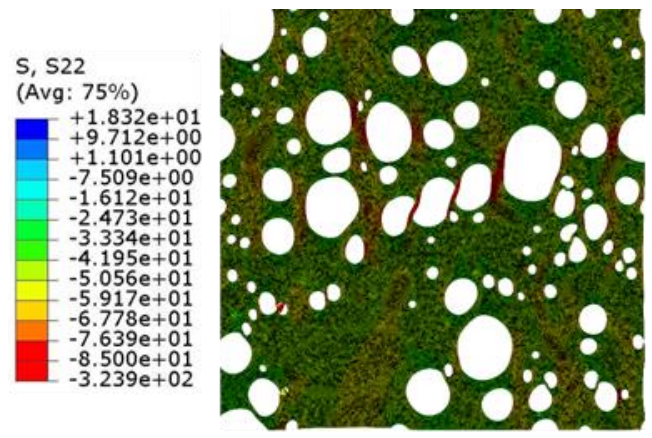

(b)

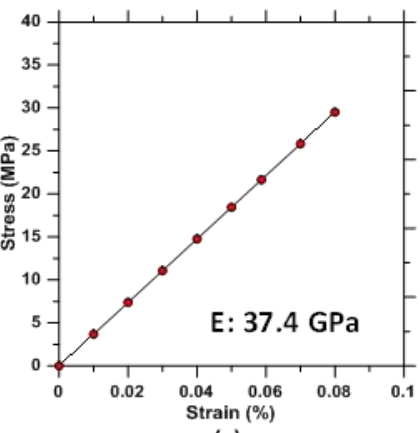

(c)

Figure 10: (a) 2D FE model showing the iron particles, pores and matrix (Size of REA: $90^{2} \mu \mathrm{m}^{2}$ ); (b) stress distribution (MPa) under an imposed strain of $0.12 \%$, (c) 2D constitutive relationship at different applied strains to determine the composite $\mathrm{E}$ 


\subsection{Microstress Distributions and Influence of Phase Properties: Comparing Fundamentally Different Binder Systems}

In addition to the material properties such as elastic modulus, it is also important to understand the stress distributions in the material under applied loading that govern material failure. This allows for strengthening/stiffening of the microstructural phases or altering their volume fractions for better composite performance. The stress distribution in the microstructure of the iron carbonate binder is compared with that in a traditional hardened cement paste in this study. $2 \mathrm{D}$ microstructures are considered here for ease of computation and comparison. Figure 11(a-1) shows the generated REA $\left(90^{2}\right.$ $\mu \mathrm{m}^{2}$ ) for the three-phase iron carbonate binder (25.8\% pores, $13.4 \%$ unreacted iron, $60.8 \%$ matrix. Figure $11(b-1)$ exhibits the generated microstructure $\left(150^{2} \mu \mathrm{m}^{2}\right)$ for hardened cement paste, for which the volume fractions of the component phases are shown in Table 2 [13]. The size of REAs are determined based on a sensitivity analysis which demonstrated negligible change in effective properties if bigger REA sizes are adopted. The other requisite input parameters including the size distribution and intrinsic mechanical properties of component phases for hardened cement paste are also reported in Table 2 [13]. The hardened cement paste corresponds to a water-to-cement ratio of 0.45 and a degree of hydration of 94.5\% [13]. The stress $\left(\sigma_{22}\right)$ distribution in the REAs are shown in Figures $11(\mathrm{a}-2)$ and $(\mathrm{b}-2)$ for iron carbonate binder and hardened cement paste respectively under an externally applied strain of $0.12 \%$.

Both the binder systems show stress concentrations in the areas where the pores are closely spaced. The average matrix stress in the iron carbonate binder is higher, attributed to the larger number of pores in this system (because of the higher porosity for this binder). The homogenized Young's modulus obtained using numerical analysis are 37.4 GPa and 23.3 GPa for the iron carbonate and hardened cement paste respectively. The quantified averaged component stresses for $0.12 \%$ externally applied strain in both the binder systems are reported in Table 3. Both matrix and inclusion (unreacted iron) in iron carbonate binder experience significantly higher stresses as compared to matrix and unhydrated cement particles in hardened cement paste at the same applied strain level. This is attributed to significantly higher stiffness of components in the iron carbonate binder than of those in hardened cement paste. The matrix in the iron carbonate binder shows about $25 \%$ higher stress as compared to the matrix in hardened cement paste. However it has been determined that the tensile strength of the iron-based binder is about 5 times higher than that of the hardened cement paste [30]. Besides, the first cracking strain and ultimate tensile strain of the iron carbonate binder are also significantly higher than those of the hardened cement paste [30]. The magnitude of stress increase in the iron carbonate binder components relative to those in hardened cement paste is insignificant considering significantly higher strength and strain capacity of the 
iron carbonate binder. However, the degree of reaction of iron in the iron-based matrix influences the properties. While for traditional cement systems, increasing degree of reaction of cement results in property enhancement due to the formation of more solid phases, the consumption of (stiffer) iron to (less stiff) reaction products, beyond a certain stage is counterproductive. This needs to be considered in designing the starting material compositions and curing conditions for the iron-based binders. Microstructural stress analysis of the type reported here can shed light into the phase amounts and properties in such unconventional binders that are designed for performance-equivalence as that of traditional binding materials.

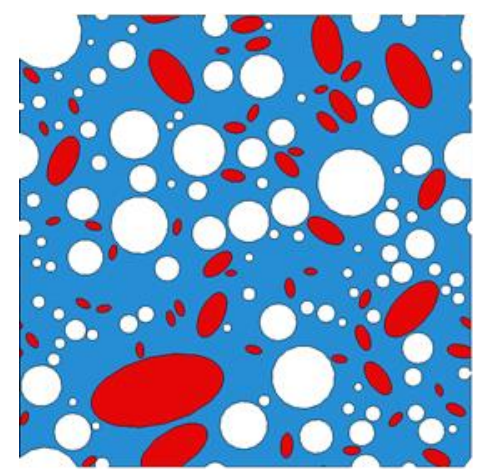

(a-1)

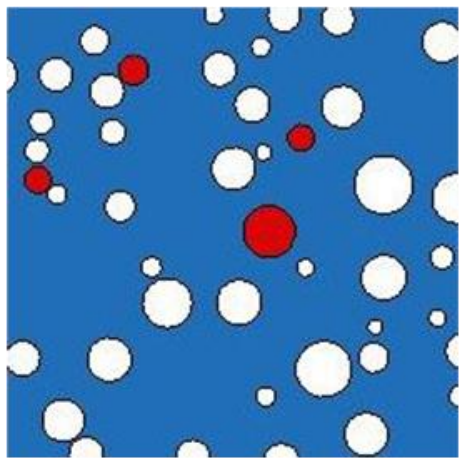

(b-1)

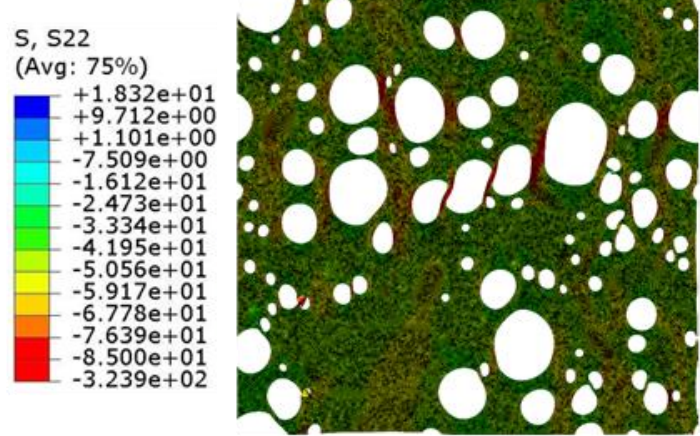

(a-2)

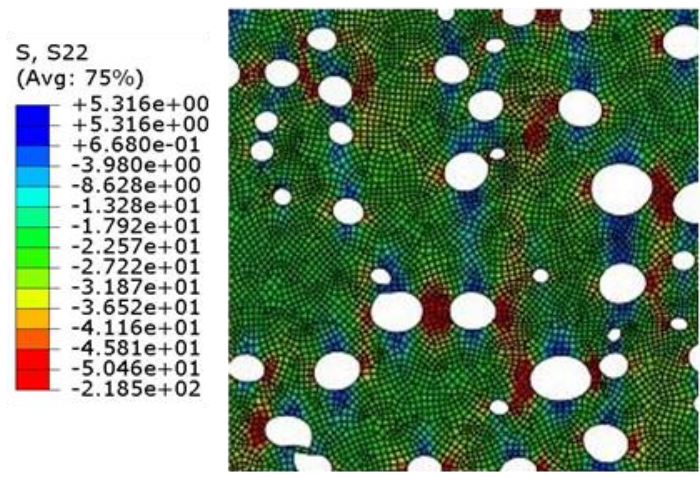

(b-2)

Figure 11: (a-1) 2D FE model for iron carbonate binder showing the iron particles (red), pores and matrix (Size of REA: $90^{2} \mu \mathrm{m}^{2}$ ); (a-2) stress distribution (MPa) in iron carbonate binder under an imposed strain of $0.12 \%$, (b-1) 2D FE model for hardened cement paste showing the Unhydrated cement particles (red), pores and matrix (Size of REA: $150^{2} \mu \mathrm{m}^{2}$ ); (b-2) stress distribution (MPa) in hardened cement paste under an imposed strain of $0.12 \%$. 
Table 2: Volume fraction, size distribution and intrinsic elastic properties of individual component phases in hardened cement paste [13]

\begin{tabular}{lcccc}
\hline Component & Volume fraction (\%) & $\mathrm{d}_{50}(\mathrm{um})$ & $\mathrm{E}(\mathrm{GPa})$ & $v$ \\
\hline Unhydrated cement & 2 & 13.2 & 132.7 & 0.3 \\
\hline Hydration product & 84 & - & 24 & 0.24 \\
\hline Pore & 14 & 10.1 & - & - \\
\hline
\end{tabular}

Table 3: Individual component stresses $\left(\sigma_{22}\right)$ in iron carbonate binder and hardened cement paste

\begin{tabular}{|c|c|c|c|c|}
\hline \multirow{2}{*}{ Binders } & \multicolumn{4}{|c|}{ Stress $\left(\sigma_{22}\right), \mathrm{MPa}$} \\
\cline { 2 - 5 } & \multicolumn{2}{|c|}{ Iron carbonate } & Hardened cement paste \\
\hline Components & Iron & Matrix & $\begin{array}{c}\text { Unhydrated } \\
\text { cement }\end{array}$ & Matrix \\
\hline Component stresses & 40.8 & 30.2 & 31 & 24.1 \\
\hline
\end{tabular}

\subsection{Fly Ash Geopolymer}

The bulk elastic response of a fly ash geopolymer is predicted using the numerical modeling scheme in conjunction with multi-scale experimentation in this section. Figure 12(a) shows a representative cubic volume of interest of the fly ash geopolymer obtained from reconstruction of synchrotron XRT images. Figure 12(b) shows the pore (red) and solid (grey) phases segmented using transition point-based thresholding coupled with the application of appropriate discrete thresholding in the microstructure to obtain the most realistic representation of known microstructural features. The segmentation procedure is described in detail in our recent work [8]. Here, the unreacted fly ash particles are not separable from partially reacted ones due to lack of sufficient absorption contrast between these phases $[8,53]$. Thus synchrotron XRT is used to quantify the pore structure alone in this study [8].

The intrinsic elastic property of component phases in the fly ash geopolymer is classified and quantified using statistical nanoindentation. The procedure is similar to that applied for the iron carbonate binder (Section 4.1.1). Figure 12(c) shows the peaks obtained through statistical deconvolution [62-64] of the histogram. Four characteristic peaks are observed in the histogram. The peaks were assigned to different solid phases based on microstructural observation. The phase with the highest elastic modulus is attributed to the unreacted fly ash present in the matrix, while the phase with the lowest elastic modulus is attributed to the N-A-S-H gel phase that is formed through diffusion of the ionic species and gel precipitation in the empty spaces in the microstructure [8]. The peak corresponding to the lower among the remaining two peaks corresponds to the fly ash particles that have reacted partially. The remaining 
peak could be attributed to particles with some reaction products on the surface, or to unreacted fly ash particles with cavities, as observed in the microstructure. The nanoindentation results are reported in detail in [8].
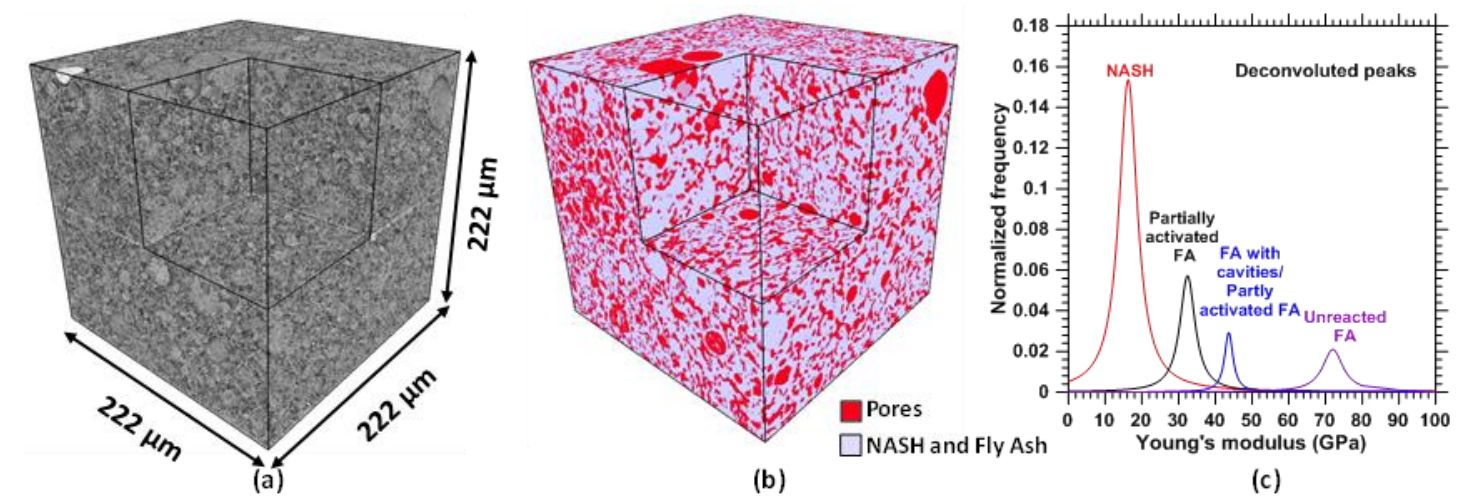

(c)

Figure 12: (a) Original XRT image, (b) 3D image after pore segmentation (two-phase; pore and solid), and (c) deconvoluted component peaks for the four distinctly identifiable microstructural phases in the solid component of the fly ash geopolymer obtained from nanoindentation. The area under each deconvoluted peak is the fraction of that respective solid phase in all solids in the paste.

For simplification, partially activated fly ash is homogenized with the N-A-S-H gel (which is the major reaction product in fly ash-based geopolymers) using the Mori-Tanaka method [6]. Furthermore, fly ash particles with cavities and the unreacted fly ash particles are homogenized together. Thus, pores, unreacted fly ash (including those with cavities) and the matrix (partially activated fly ash homogenized with the N-A-S-H gel) are used as the different microstructural phases in numerical simulation. The size distribution of unreacted fly ash (starting material) as obtained from laser diffraction [8] is used in numerical simulation since unreacted fly ash particles could not be segmented separately from partially activated fly ash in XRT for reasons explained earlier.

Two-stage numerical homogenization is performed to obtain homogenized Young's modulus of the geopolymer. In Stage-I, the unreacted fly ash particles are homogenized with the matrix. Figure 13(a) shows the 3D microstructure containing fly ash (green) and the matrix (blue) phases. The RVE is meshed with tetrahedral C3D10 elements (in ABAQUS ${ }^{\mathrm{TM}}$ ) and periodic boundary conditions are implemented as explained earlier. Figure 13(b) shows the dominant principal stress distribution in the RVE corresponding to an externally applied strain of $0.12 \%$. The constitutive relationship thus obtained after the application of different strains is shown in Figure 13(c). In Stage-II, the homogenized value from Stage-I is used as the elastic modulus of the matrix, and the pores are incorporated into the microstructure (Figure 14(a)). 
Figure 14(b) shows the dominant principal stress distribution in the unit cell when an externally applied strain of $0.1 \%$ is applied. The constitutive relationship for the fly ash geopolymer is shown in Figure 14 (c). The numerically homogenized value of 15.6 GPa correlates well with the experimentally obtained Young's modulus of $14.73 \pm 0.7 \mathrm{GPa}[8]$.

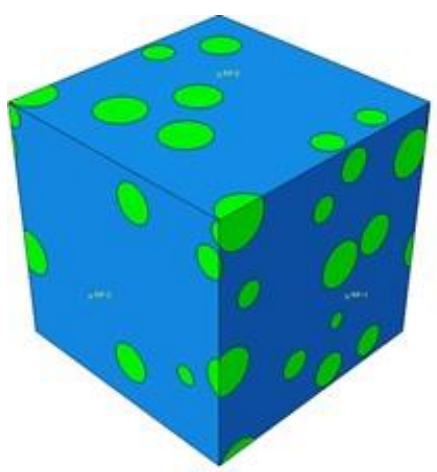

(a)

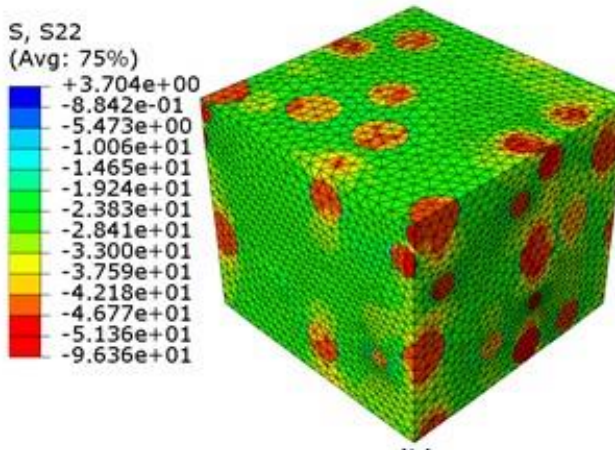

(b)

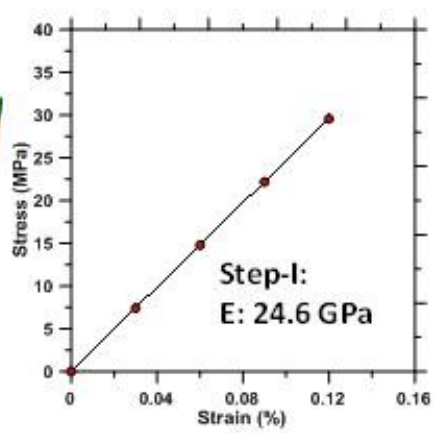

(c)

Figure 13: Step-I: (a) FE model showing the unreacted fly ash and matrix; (b) stress distribution (MPa) under an imposed strain of $0.12 \%$ which results in an average RVE stress of $29.52 \mathrm{MPa}$, and (c) the constitutive relationship in the linear elastic regime.

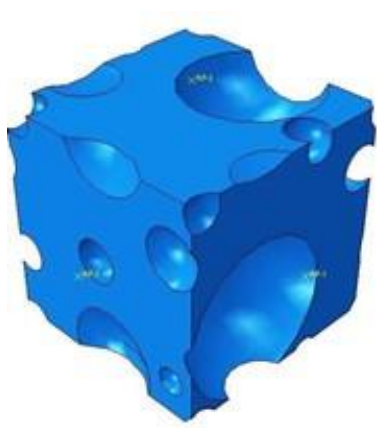

(a)

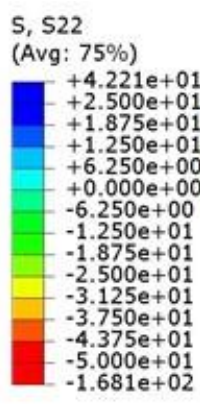

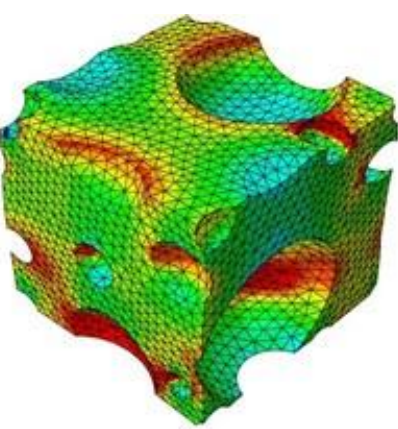

(b)

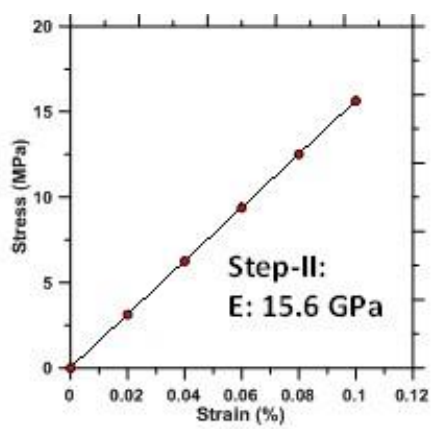

(c)

Figure 14: Stage-II: (a) FE model showing the matrix (from Stage-I) and the pores ; (b) stress distribution $(\mathrm{MPa})$ under an imposed strain of $0.1 \%$ which results in an average RVE stress of $15.6 \mathrm{MPa}$, and $(\mathrm{c})$ the constitutive relationship in the linear elastic regime.

\section{SUMMARY AND CONCLUSIONS}

This paper presents a comprehensive numerical approach that derives inputs from high-fidelity experiments towards the prediction of effective elastic response of heterogeneous binding materials. The applicability of the approach is confirmed through its successful implementation on two fundamentally different heterogeneous binding media - a densified iron carbonate binder, and a fly ash geopolymer. Synchrotron XRT information was used to reliably estimate the pore (and particle, in some cases) volume 
and size distribution in 3D, while nanoindentation was used to determine the intrinsic elastic properties of the microstructural phases which served as inputs to the numerical micromechanical scheme. 3D microstructures were generated using the Lubachhevsky-Stillinger algorithm, and periodic boundary conditions implemented. The microstructural information and the constraint equations to implement periodic boundary conditions were integrated into a python script to facilitate numerical implementation of a displacement controlled test in the FE environment.

The iron carbonate binder displayed an extremely high degree of microstructural heterogeneity, with the reaction product comprising of solid phases of varying density, in addition to the unreacted iron particles and the pore phase. The fly ash geopolymer also demonstrated heterogeneity, though not to the extent of the iron carbonate binder. This necessitated a step-wise homogenization scheme to be implemented prior to the numerical modeling in order to limit the computational time and expense. The solid phases that showed lower contrast in their elastic moduli were sequentially homogenized using analytical schemes such as the Mori-Tanaka method to obtain the elastic moduli of the matrix phase in both the binders. The matrix and the unreacted iron in the iron carbonate binder or the unreacted fly ash in the geopolymer were subjected to 3D FE analysis in the first stage, and the numerically homogenized matrix along with the pore phase were analyzed in the second stage to obtain the composite elastic modulus. The elastic moduli obtained from numerical simulation matched well with those from macroscale experiments for both the binders. A 2D model containing all the three microstructural phases (pores, unreacted particles, and the matrix) was also implemented with satisfactory results.

The validation of the methodology presented here provides confidence on its ability to be applied for many classes of highly heterogeneous multi-phase materials. In addition to predicting the composite elastic modulus, this method allows for the prediction of stresses in the individual components of the microstructure under varying applied external loads (or strains), thereby providing an understanding of probable failure modes and help design strategies to tailor the microstructure for requisite mechanical performance.

\section{ACKNOWLEDGEMENTS}

The authors gratefully acknowledge the National Science Foundation (NSF) for the support for this research (CMMI: 1068985 and CMMI: 1353170). This research was conducted in the Laboratory for the Science of Sustainable Infrastructural Materials, the Structural Engineering Laboratory, 4D Materials Science Laboratory, and LeRoy Eyring Center for Solid State Science at Arizona State University and the 
supports that have made these laboratories possible are acknowledged. The 2-BM beamline of the Advanced Photon Source (APS) at Argonne National Laboratory is also acknowledged. The contents of this paper reflect the views of the authors who are responsible for the facts and accuracy of the data presented herein, and do not necessarily reflect the views and policies of the funding agency, nor do the contents constitute a standard, specification, or a regulation.

\section{REFERENCES}

[1] P. Sheng, Phys. Rev. B 41 (1990) 4507-4512.

[2] Z. Sun, E.J. Garboczi, S.P. Shah, Cem. Concr. Compos. 29 (2007) 22-38.

[3] S. Mercier, A. Molinari, Int. J. Plast. 25 (2009) 1024-1048.

[4] J. Segurado, R.A. Lebensohn, J. LLorca, C.N. Tomé, Int. J. Plast. 28 (2012) 124-140.

[5] R.A. Lebensohn, C.N. Tomé, P.P. CastaÑeda, Philos. Mag. 87 (2007) 4287-4322.

[6] T. Mori, K. Tanaka, Acta Metall. 21 (1973) 571-574.

[7] M. Hori, S. Nemat-Nasser, Mech. Mater. 14 (1993) 189-206.

[8] S. Das, P. Yang, S.S. Singh, J.C.E. Mertens, X. Xiao, N. Chawla, N. Neithalath, Cem. Concr. Res. 2015 (2015).

[9] C.F. Dunant, B. Bary, A.B. Giorla, C. Péniguel, J. Sanahuja, C. Toulemonde, A.-B. Tran, F. Willot, J. Yvonnet, Adv. Eng. Softw. 58 (2013) 1-12.

[10] M.I. Idiart, F. Willot, Y.-P. Pellegrini, P. Ponte Castañeda, Int. J. Solids Struct. 46 (2009) 3365-3382.

[11] V.P. Nguyen, M. Stroeven, L.J. Sluys, Comput. Methods Appl. Mech. Eng. 201-204 (2012) 139-156.

[12] J.L. Zhang, X. Liu, Y. Yuan, H.A. Mang, Comput. Mech. 55 (2014) 37-55.

[13] M. Hain, P. Wriggers, Comput. Mech. 42 (2007) 197-212.

[14] J. Sanahuja, C. Toulemonde, Cem. Concr. Res. 41 (2011) 1320-1329.

[15] J. Paiboon, D.V. Griffiths, J. Huang, G.A. Fenton, Int. J. Solids Struct. 50 (2013) 3233-3241.

[16] N. Chawla, B.V. Patel, M. Koopman, K.K. Chawla, R. Saha, B.R. Patterson, E.R. Fuller, S.A. Langer, Mater. Charact. 49 (2002) 395-407.

[17] N. Chawla, V.V. Ganesh, B. Wunsch, Scr. Mater. 51 (2004) 161-165.

[18] R.S. Sidhu, N. Chawla, Scr. Mater. 54 (2006) 1627-1631.

[19] N. Chawla, K.K. Chawla, J. Mater. Sci. 41 (2006) 913-925.

[20] E. Padilla, V. Jakkali, L. Jiang, N. Chawla, Acta Mater. 60 (2012) 4017-4026.

[21] I. Comby-Peyrot, F. Bernard, P.-O. Bouchard, F. Bay, E. Garcia-Diaz, Comput. Mater. Sci. 46 (2009) 1163-1177.

[22] C.F. Dunant, K.L. Scrivener, Cem. Concr. Res. 40 (2010) 517-525.

[23] J. Escoda, F. Willot, D. Jeulin, J. Sanahuja, C. Toulemonde, Cem. Concr. Res. 41 (2011) 542-556.

[24] M. Hain, P. Wriggers, Finite Elem. Anal. Des. 44 (2008) 233-244.

[25] K. Terada, M. Hori, T. Kyoya, N. Kikuchi, Int. J. Solids Struct. 37 (2000) 2285-2311.

[26] O. van der Sluis, P.J.G. Schreurs, W.A.M. Brekelmans, H.E.H. Meijer, Mech. Mater. 32 (2000) 449462.

[27] S. Das, B. Souliman, D. Stone, N. Neithalath, ACS Appl. Mater. Interfaces 6 (2014) 8295-8304.

[28] S. Das, A. Hendrix, D. Stone, N. Neithalath, Constr. Build. Mater. 93 (2015) 360-370.

[29] S. Das, D. Stone, D. Convey, N. Neithalath, Mater. Charact. 98 (2014) 168-179.

[30] S. Das, D. Stone, B. Mobasher, N. Neithalath, Eng. Fract. Mech. 156 (2016) 1-15.

[31] S. Das, A. Kizilkanat, S. Chowdhury, D. Stone, N. Neithalath, Mater. Des. 92(2016) 189-199.

[32] D. Ravikumar, S. Peethamparan, N. Neithalath, Cem. Concr. Compos. 32 (2010) 399-410.

[33] M. Chi, R. Huang, Constr. Build. Mater. 40 (2013) 291-298. 
[34] B.D. Lubachevsky, F.H. Stillinger, J. Stat. Phys. 60 (1990) 561-583.

[35] B.D. Lubachevsky, J. Comput. Phys. 94 (1991) 255-283.

[36] B.D. Lubachevsky, F.H. Stillinger, E.N. Pinson, J. Stat. Phys. 64 (1991) 501-524.

[37] H.A. Meier, E. Kuhl, P. Steinmann, Int. J. Numer. Anal. Methods Geomech. 32 (2008) 509.

[38] P. Wriggers, S.O. Moftah, Finite Elem. Anal. Des. 42 (2006) 623-636.

[39] C. Huet, Mech. Mater. 31 (1999) 787-829.

[40] M. Ostoja-Starzewski, Mech. Mater. 31 (1999) 883-893.

[41] S. Hazanov, C. Huet, J. Mech. Phys. Solids 42 (1994) 1995-2011.

[42] S. Hazanov, Arch. Appl. Mech. 68 (1998) 385-394.

[43] P. Suquet, Elements of homogenization for inelastic solid mechanics (1987).

[44] S. Li, Compos. Sci. Technol. 68 (2008) 1962-1974.

[45] Z. Xia, C. Zhou, Q. Yong, X. Wang, Int. J. Solids Struct. 43 (2006) 266-278.

[46] C.T. Sun, R.S. Vaidya, Compos. Sci. Technol. 56 (1996) 171-179.

[47] B.M. Patterson, N.L. Cordes, K. Henderson, J.J. Williams, T. Stannard, S.S. Singh, A.R. Ovejero, X. Xiao, M. Robinson, N. Chawla, J. Mater. Sci. 51 (2015) 171-187.

[48] J.J. Williams, Z. Flom, A.A. Amell, N. Chawla, X. Xiao, F. De Carlo, Acta Mater. 58 (2010) 6194-6205.

[49] F. De Carlo, B. Tieman, in:, 2004, pp. 644-651.

[50] B.A. Dowd, G.H. Campbell, R.B. Marr, V.V. Nagarkar, S.V. Tipnis, L. Axe, D.P. Siddons, in:, 1999, pp. 224-236.

[51] M.L. Rivers, in:, 2012, p. 85060U-85060U-13.

[52] F. Marone, M. Stampanoni, J. Synchrotron Radiat. 19 (2012) 1029-1037.

[53] J.L. Provis, R.J. Myers, C.E. White, V. Rose, J.S.J. van Deventer, Cem. Concr. Res. 42 (2012) 855-864.

[54] N. Bossa, P. Chaurand, J. Vicente, D. Borschneck, C. Levard, O. Aguerre-Chariol, J. Rose, Cem. Concr. Res. 67 (2015) 138-147.

[55] M. Zhang, Y. He, G. Ye, D.A. Lange, K. van Breugel, Constr. Build. Mater. 27 (2012) 472-481.

[56] P.J.M. Monteiro, A.P. Kirchheim, S. Chae, P. Fischer, A.A. MacDowell, E. Schaible, H.R. Wenk, Cem. Concr. Compos. 31 (2009) 577-584.

[57] E.J. Garboczi, D.P. Bentz, Cem. Concr. Res. 31 (2001) 1501-1514.

[58] W. C. Oliver, G. M. Pharr, J. Mater. Res. 19 (2004) 3-20.

[59] X. Li, B. Bhushan, Mater. Charact. 48 (2002) 11-36.

[60] N.R. Moody, W.W. Gerberich, N. Burnham, S.P. Baker, Fundamentals of Nanoindentation and Nanotribology, Warrendale, PA (United States); Materials Research Society, 1998.

[61] L. Sorelli, G. Constantinides, F.-J. Ulm, F. Toutlemonde, Cem. Concr. Res. 38 (2008) 1447-1456.

[62] W.R.L. da Silva, J. Němeček, P. Štemberk, Adv. Eng. Softw. 62-63 (2013) 109-118.

[63] G. Constantinides, K.S. Ravi Chandran, F.-J. Ulm, K.J. Van Vliet, Mater. Sci. Eng. A 430 (2006) 189202.

[64] J. Němeček, V. Šmilauer, L. Kopecký, Cem. Concr. Compos. 33 (2011) 163-170.

[65] A. Reuss, Z Angew Math Mech 9 (1929) 49-58.

[66] W. Voigt, Z Angew Math Mech 9 (1929) 49.

[67] C.-J. Haecker, E.J. Garboczi, J.W. Bullard, R.B. Bohn, Z. Sun, S.P. Shah, T. Voigt, Cem. Concr. Res. 35 (2005) 1948-1960.

[68] M. Kamiński, M. Kazimierczak, Compos. Struct. 108 (2014) 1009-1018.

[69] Q. Yao, J. Qu, J. Electron. Packag. 121 (1999) 196-201. 